\title{
Un caso de hipofosfatasia perinatal grave con una mutación novedosa
}

\author{
Severe perinatal hypophosphatasia case with a novel mutation
}

Havva Yazicia (D), Ebru Canda ${ }^{a}$ (D), Sema Kalkan Ucar ${ }^{a}$ (D), Mahmut Coker ${ }^{a}$ (D)

\begin{abstract}
RESUMEN
La hipofosfatasia es un trastorno hereditario raro causado por mutaciones en el gen ALPL. Causa defectos en la mineralización ósea y dental, función respiratoria anormal, convulsiones, hipotonía, dolor óseo y nefrocalcinosis. Las formas clínicas se reconocen según la edad al diagnóstico y la gravedad.

Presentamos el caso de una lactante con fontanela anterior agrandada, bóveda craneal blanda, fracturas, dificultad respiratoria y convulsiones. El análisis bioquímico mostró hipercalcemia, fosfato sérico normal y fosfatasa alcalina sérica baja. La radiografía mostró hipomineralización, fracturas y callos. La concentración plasmática de piridoxal-5'-fosfato era de $762 \mathrm{mg} / 1$ (intervalo normal: 5-50) y la concentración de fosfoetanolamina en orina era de $1015 \mathrm{mmol} / 1$ (intervalo normal: 15-341). El análisis del gen ALPL mostró dos mutaciones heterocigotas compuestas, una de las cuales es novedosa. El diagnóstico y tratamiento tempranos de la hipofosfatasia perinatal podría mejorar los resultados y tener un impacto positivo en la sobrevida.

Palabras clave: hipofosfatasia, gen ALPL, asfotasaalfa, tratamiento de reemplazo enzimático.
\end{abstract}

http: / / dx.doi.org/10.5546/ aap.2022.e21

Texto completo en inglés:

http: / / dx.doi.org/ 10.5546/ aap.2022.eng.e21

Cómo citar: Yazici H, Canda E, Kalkan Ucar S, Coker M. Un caso de hipofosfatasia perinatal grave con una mutación novedosa. Arch Argent Pediatr 2022;120(1):e21-e24.

\section{INTRODUCCIÓN}

La hipofosfatasia es una enfermedad rara del metabolismo óseo y mineral causada por la baja actividad de la isoenzima tisular inespecífica de la fosfatasa alcalina (TNSALP, por sus siglas en inglés) causada por mutaciones en el gen que codifica la TNSALP.

a. Departamento de Metabolismo y Nutrición en Pediatría, Facultad de Medicina de Ege Üniversitesi, Esmirna, Turquía.

Correspondencia: Havva Yazici: havvaya@gmail.com

Financiamiento: Ninguno.

Conflicto de intereses: Ninguno que declarar.

Recibido: 9-12-2020

Aceptado: 13-7-2021
En 1923, se describió la FA por primera vez. El primer caso de hipofosfatasia se informó en $1948 .{ }^{1}$ La hipofosfatasia refleja la actividad defectuosa de la TNSALP. La actividad reducida de la FA sérica es la primera característica bioquímica principal y de fácil detección de la hipofosfatasia. Existen solo unos pocos estudios que informan sobre la incidencia y la prevalencia de la hipofosfatasia. Las formas de aparición temprana (perinatal, infantil) de la hipofosfatasia ocurren en aproximadamente 1 cada 100000 y 300000 nacimientos en Canadá y Europa, respectivamente. ${ }^{2}$ El espectro clínico varía notablemente: de muerte neonatal a problemas dentales que se manifiestan con o sin osteopatía en la adultez. Se describen las siguientes formas clínicas, que se clasifican según la edad al momento de aparición de la hipofosfatasia: hipofosfatasia "adulta", "pediátrica", "infantil" y "perinatal". Las manifestaciones clínicas de la hipofosfatasia perinatal in utero son similares a otras displasias esqueléticas. Las características clínicas congénitas son cráneo blando, extremidades acortadas, deformidad torácica y muerte fetal. Otros hallazgos clínicos en el período neonatal incluyen apnea, insuficiencia respiratoria, convulsiones dependientes de vitamina B6 y fracturas. ${ }^{1-3}$

Hasta muy recientemente, la hipofosfatasia perinatal grave era mortal al momento del nacimiento o en los primeros meses de vida. La asfotasa alfa (STRENSIQ ${ }^{\circledR}$, Alexion Pharmaceuticals, Inc., Boston, MA, EE. UU.) es un tratamiento de reemplazo enzimático recombinante humano óseo que se administra por vía subcutánea. Está aprobada para el tratamiento de los pacientes con hipofosfatasia y mejoró la mineralización ósea, la sobrevida y la sobrevida sin ventilación. ${ }^{1,4,5}$ La hipofosfatasia perinatal debe considerarse en el diagnóstico diferencial de los pacientes con deformidades esqueléticas, convulsiones, hipercalcemia y concentración baja de FA. El diagnóstico y tratamiento tempranos de la hipofosfatasia perinatal podría mejorar los resultados y tener un impacto positivo en la sobrevida. 
Esta es la presentación clínica de un caso de hipofosfatasia diagnosticada en el período neonatal.

\section{A PROPÓSITO DE UN CASO}

Nació una niña sana de padres no consanguíneos a las 38 semanas de gestación por cesárea. Su peso al nacer fue de $2800 \mathrm{~g}$. En el examen inicial, se observó fontanela anterior agrandada, bóveda craneal blanda, tórax estrecho y en forma de campana y ambos antebrazos arqueados. Presentaba taquipnea, tiraje subcostal y quejidos espiratorios. Requirió presión positiva continua nasal durante cinco días, pero luego no necesitó oxigenoterapia. El día 6 tuvo una convulsión. Se le hizo un electroencefalograma, que detectó un patrón paroxístico y actividad epiléptica multifocal. Recibió tratamiento con fenitoína intravenosa en una dosis de carga de $20 \mathrm{mg} / \mathrm{kg}$ y una dosis de mantenimiento de $5 \mathrm{mg} / \mathrm{kg}$. No tuvo convulsiones los días posteriores.

El hemograma completo fue normal. El análisis bioquímico mostró una concentración sérica baja de FA (10 U/1, normal: > 70). En la Tabla 1 se presentan los intervalos de referencia de la FA por edad. La concentración sérica de calcio era elevada (13,5 mg/ dl; intervalo normal: 9-10,9) y la concentración sérica de fosfato era normal $(4,9 \mathrm{mg} /$ dl; intervalo normal: 3,4-5,9). La concentración sérica de 25-hidroxivitamina D3 era normal (96 nmol/l; el valor de corte para la deficiencia de vitamina D3 es $<25$ ) y la hormona paratiroidea era baja ( $3 \mathrm{pg} / \mathrm{ml}$; intervalo normal: 11-67). El cociente calcio/creatinina en la muestra puntual de orina era normal $(0,6 \mathrm{mg} / \mathrm{mg}$; normal: $<0,8)$.

En las radiografías óseas se observaron estructuras óseas radiotransparentes y costillas

TABLA 1. Intervalos de referencia de la concentración de fosfatasa alcalina (U/l) por edad ${ }^{6}$

\begin{tabular}{lcc}
\hline Edad & Sexo masculino & Sexo femenino \\
\hline$<1$ mes & 60 & 60 \\
$1-11$ meses & 70 & 70 \\
$1-3$ años & 125 & 125 \\
$4-11$ años & 150 & 150 \\
$12-13$ años & 160 & 110 \\
$14-15$ años & 60 & 40 \\
$\geq 20$ años & 40 & 40 \\
\hline
\end{tabular}

delgadas. También se detectaron fracturas del radio y el cúbito y formaciones callosas. La ecografía de cráneo fue normal. Se observó nefrocalcinosis bilateral en la ecografía abdominal a los 14 días de vida. La tomografía del cráneo fue normal.

La concentración plasmática de piridoxal5'-fosfato (PLP, por su sigla en inglés) y la concentración de fosfoetanolamina (PEA, por su sigla en inglés) en orina eran elevadas: 762 ug/l (normal: 5-50) y $1015 \mathrm{umol} / 1$ (normal: 15-341), respectivamente. El diagnóstico presunto era de hipofosfatasia perinatal grave, confirmado por la determinación bioquímica de PLP y PEA. El análisis del gen ALPL mostró heterocigosis compuesta en p.R184W (c.550C > T)/ p.G288A (c.863G> C).

La paciente recibió tratamiento con asfotasa alfa por vía subcutánea en una dosis de $2 \mathrm{mg} / \mathrm{kg}$ por primera vez a los 60 días de vida. El tratamiento continuó tres veces a la semana. Las primeras dos dosis de asfotasa alfa se administraron en el hospital, sin reacción en el lugar de aplicación. Tampoco se observaron otros eventos adversos (hipocalcemia, hiperfosfatemia y calcificación) en nuestra paciente. Se recomendó utilizar alimentación nasogástrica debido a la hipotonicidad de la paciente, pero su familia se rehusó. La paciente recibió el alta a los 64 días de vida. Después de la quinta dosis de asfotasa alfa subcutánea, a los 72 días de vida, los padres hallaron a la niña en su cama; no respondía a los estímulos ni respiraba. La familia acudió al centro de salud más cercano y en el instituto sanitario declararon el fallecimiento de la paciente. No fue posible realizar una autopsia porque la familia no otorgó la autorización legal necesaria. Por lo tanto, no pudimos obtener datos sobre un evento de aspiración, que podría haber causado la muerte en un lactante hipotónico.

\section{DISCUSIÓN}

La hipofosfatasia perinatal es la forma más grave de la enfermedad. En nuestra paciente, se detectaron fracturas y callos en los primeros días de vida, lo que indica que la afección comenzó en el período intrauterino. También se observó dificultad respiratoria los primeros días de vida. La hipofosfatasia perinatal está asociada con mortalidad significativa y debe distinguirse de la forma "prenatal benigna", que se refiere a una deformidad esquelética in utero o nacimiento ex utero que mejora espontáneamente fuera del útero. ${ }^{1}$ 
Nuestra paciente requirió asistencia respiratoria durante cinco días. La mortalidad es elevada, entre el $50 \%$ y el $100 \%$ en la hipofosfatasia perinatal, como consecuencia de la insuficiencia respiratoria causada por la hipoplasia pulmonar y los defectos funcionales secundarios a la deformidad del tórax. . $^{1,4,5,7}$

En el caso presentado aquí, las deformidades esqueléticas, una concentración baja de FA, hipercalcemia, una concentración sérica normal de 25-hidroxivitamina D3 y una concentración baja de hormona paratiroidea fueron fuertes indicadores de hipofosfatasia. La concentración elevada de PLP en plasma $y$ de PEA en orina es el rasgo bioquímico característico de este caso. Aunque no se midió en esta paciente, el pirofosfato inorgánico (PPi) es uno de los inhibidores más significativos de la mineralización.

El diagnóstico diferencial incluye raquitismo, síndrome de inatención unilateral, traumatismo no accidental, osteogénesis imperfecta de tipo II, displasia tanatofórica, displasia campomélica y condrodisplasias con defectos en la mineralización ósea. ${ }^{1,3,6}$

Las convulsiones dependientes de la piridoxina suelen ser un indicador pronóstico fatal. El PLP es una de las formas intracelulares bioactivas significativas de la vitamina B6. Si no es posible desfosforilar el PLP a piridoxal (PL) para que cruce la barrera hematoencefálica, podrían ocurrir convulsiones dependientes de la vitamina B6. ${ }^{1,3,5}$

En nuestra paciente, fue posible controlar las convulsiones sin necesidad de usar piridoxina.

Puede observarse nefrolitiasis a causa de hipercalcemia o hipercalciuria. ${ }^{1,3,4}$ En nuestra paciente, la ecografía abdominal reveló nefrocalcinosis bilateral. Nuestra paciente también tenía hipercalcemia.

En el análisis del gen $A L P L$, se observó una mutación heterocigota compuesta: p.R184W (c.550C>T)/p.G288A (c.863G>C). La mutación p.R184W (c.550C $>$ T) está asociada experimentalmente con un $0,6 \%$ de la actividad enzimática natural, lo que es pronóstico de un fenotipo grave. ${ }^{1,7-9}$ Hasta donde sabemos, este es el primer informe sobre la mutación p.G288A (c.863G $>$ C). Tal expresividad se explica mayormente por la transmisión autosómica recesiva (AR) frente a la transmisión autosómica dominante $(\mathrm{AD})$ de, al menos, 275 mutaciones dentro del gen $A L P L$, de las cuales, el $74 \%$ es de sentido erróneo. La herencia AR suele explicar la hipofosfatasia grave, mientras que la herencia tanto AD como AR se relaciona con hipofosfatasia leve. ${ }^{3}$ Se analizó a los padres mediante secuenciación dirigida y se confirmaron dos variantes en el gen ALPL. Las concentraciones de FA de su madre y su padre eran normales: 86 $\mathrm{U} / 1$ y $73 \mathrm{U} / 1$, respectivamente.

La primera opción terapéutica con medicamentos es el tratamiento de reemplazo enzimático óseo; la asfotasa alfa se ha indicado para el tratamiento de reemplazo enzimático a largo plazo en los pacientes con hipofosfatasia pediátrica para tratar las manifestaciones óseas de la enfermedad. ${ }^{1,5,7} \mathrm{El}$ tratamiento de reemplazo enzimático puede mejorar la sobrevida. En 2016, Whyte y col., informaron una mejor sobrevida en los pacientes con hipofosfatasia perinatal e infantil tratados frente a los controles históricos: $95 \%$ frente al $42 \%$ al año de edad y $84 \%$ frente al $27 \%$ a los 5 años de edad, respectivamente. ${ }^{4}$

En los ensayos clínicos, también se informaron la seguridad y la mejoría de la mineralización y la función respiratoria. ${ }^{4,5,7}$

El evento adverso más frecuente del tratamiento con asfotasa alfa fue la reacción en el lugar de la aplicación de la inyección. Nuestra paciente no presentó reacciones locales. Tampoco se observaron otros eventos adversos, como hipocalcemia, hiperfosfatemia y calcificación ectópica. ${ }^{4,5,7}$

Se debe incluir la hipofosfatasia congénita en el diagnóstico diferencial en presencia de hallazgos en varios aparatos y sistemas, como deformidad esquelética, fracturas espontáneas, hipotonía, dificultad respiratoria y convulsiones. Se informaron efectos positivos sobre la mineralización ósea a las cuatro semanas de iniciar el tratamiento, y puede observarse una mejoría en la función respiratoria luego de varios meses. ${ }^{4,5,7}$ En nuestra paciente, la duración del tratamiento no fue suficiente para evaluar la respuesta ósea y pulmonar.

Nuevamente, este caso destaca la importancia de iniciar el tratamiento tan pronto como sea posible tras el nacimiento. Por último, un diagnóstico temprano de la hipofosfatasia perinatal, el reconocimiento prenatal y el inicio del tratamiento de reemplazo enzimático tan pronto como sea posible después del nacimiento podrían mejorar los resultados y tener un impacto positivo en la sobrevida. Asimismo, hasta donde sabemos, la mutación p.G288A establecida en nuestra paciente es una alteración identificada recientemente. 


\section{Nota aclaratoria}

Los autores declaran que no tienen ninguna relación económica relevante respecto de este artículo.

Alexion Pharmaceuticals Inc. hizo una revisión médica/ científica de la presentación del caso como gentileza a pedido de los autores; estos tuvieron la decisión final sobre todo el contenido de esta publicación.

\section{REFERENCIAS}

1. Whyte MP.Hypophosphatasia: an overview for 2017. Bone. 2017; 102:15-25.

2. Vogt M, GirschickH, Schweitzer T, Benoit C, et al. Pediatric hypophosphatasia: lessons learned from a retrospective single-center chart review of 50 children. Orphanet J Rare Dis. 2020; 15(1):212.

3. Whyte MP, Zhang F, Wenkert D, McAlister WH, et al. Hypophosphatasia: validation and expansion of the clinical nosology for children from 25 years experience with 173 pediatric patients. Bone. 2015; 75:229-39.
4. Whyte MP, Rockman-Greenberg C, Ozono K, Riese R, et al. Asfotase alfa treatment improves survival for perinatal and infantile hypophosphatasia. J Clin Endocrinol Metab. 2016; 101(1):334-42.

5. Kitaoka T, Tajima T, Nagasaki K, Kikuchi T, et al. Safety and efficacy of treatment with asfotase alfa in patients with hypophosphatasia: results from a Japanese clinical trial. Clin Endocrinol (Oxf). 2017; 87(1):10-9.

6. Simmons J. Best practices in: recognizing and diagnosing hypophosphatasia. Clinical Endocrinology News. 2013. [Fecha de acceso: 6 de julio de 2021]. Disponible en: https:// www.mdedge.com/endocrinology/article/78038/ bestpractices-recognizing-and-diagnosing-hypophosphatasia

7. Scott LJ. Asfotase alfa in perinatal/infantile-onset and juvenile-onset hypophosphatasia: a guide to its use in the USA. BioDrugs. 2016; 30(1):41-8.

8. Mornet E, Taillandier A, Peyramaure S, Kaper F, et al. Identification of fifteen novel mutations in the tissuenonspecific alkaline phosphatase (TNSALP) gene in European patients with severe hypophosphatasia. Eur J Hum Genet. 1998; 6(4):308-14.

9. Sağlam H, Erdöl Ş, Dorum S. Clinical and genetic findings of Turkish hypophosphatasia cases. J Clin Res Pediatr Endocrinol. 2017; 9(3):229-36. 